a sunbarrier preparation containing 5-methoxypsoralen and suggested that once psoralen had provoked a tan there might be improved protection from further damage from solar ultraviolet radiation. This might be especially important to those Australians whose skin tans poorly. At present, therefore, opinion is somewhat divided about the balance between risk and benefit for 5-methoxypsoralen as an ingredient of suntan preparations. In the case of PUVA treatment for severe psoriasis or vitiligo, however, the consensus view remains that the benefits far outweigh the risk of skin cancer.

Much research remains to be done on this subject. The mechanisms whereby psoralens cause photosensitivity, mutagenesis, and carcinogenesis are not clear. At the molecular level two types of photoadduct lesions with DNA have been described, one in which the psoralen is covalently bonded to only one DNA strand and another where the two strands of the duplex are cross-linked. Natural repair of psoralen-DNA lesions is probably much less well developed in rodents that in man. Hence the results of mouse studies with psoralens may be misleading. The cross-linking psoralen DNA lesion interferes with DNA synthesis-which may be how PUVA works in psoriasis-and this cross-linking DNA lesion has been thought to be more lethal, more mutagenic, and more carcinogenic than the single-strand lesion. Another view, however, is that because of its lethality the cross-linking DNA lesion is less likely to give rise to mutant cells or cancer. These conflicting views need to be resolved. Certain psoralens, such as angelicin, through steric hindrance form photoadducts binding only one DNA spiral, whereas other psoralens such as 8- and 5methoxypsoralen cause cross-links. If a "safe" psoralennatural or synthetic-could be identified it could replace 8methoxypsoralen in the PUVA treatment of psoriasis. And a "safe" psoralen which provoked tanning would be a highly desirable ingredient of sunbarrier preparations.

1 Stern RS, Thibodeau LA, Kleinerman RA, Parrish JA, Fitzpatrick TB. Risk of cutaneous carcinoma in patients treated with oral methoxsalen photochemotherapy for psoriasis. N Englf Med 1979;300:809-13.

2 Ashwood-Smith MJ, Poulton GA, Barker M, Mildenberger M. 5-Methoxypsoralen, an ingredient in several suntan preparations, has lethal, mutagenic and clastogenic properties. Nature $1980 ; 285: 407-9$.

${ }^{3}$ Zajdela F, Bisagni E. 5-Methoxypsoralen, the melanogenic additive in sun-tan preparations, is tumorigenic in mice exposed to $365 \mathrm{~nm} \mathrm{UV}$ radiation. Carcinogenesis $1981 ; 2: 121-7$.

\section{Drug-resistant tuberculosis}

Drug-resistant strains of Mycobacterium tuberculosis emerge whenever one of the antituberculosis drugs is given alone to heavily infected patients. Mutant bacilli are always present as a small proportion of the original bacterial population, and these overgrow sensitive organisms during treatment. This process was recognised very soon after streptomycin was first used in the treatment of tuberculosis ${ }^{1}$ and led to the policy of using one or more additional drugs to inhibit the growth of the naturally resistant strains. The efficiency with which a drug prevents resistance from emerging depends on whether it can inhibit the growth of all the bacilli in the lesions all of the time. Rifampicin and streptomycin are the most valuable drugs in preventing the emergence of drug resistance, while pyrazinamide is also useful because of its sterilising action. ${ }^{2}$

Attention to the importance of drug-resistant tuberculosis has recently been drawn by Aswapokee and colleagues ${ }^{3}$ from New York City and by the publication of a world atlas of initial drug resistance by the International Union Against Tuberculosis. ${ }^{4}$ The union's committee on bacteriology and immunology acknowledges a major problem with the reliability of its data because susceptibility testing of $M$ tuberculosis requires a high standard of laboratory skill, which varies from country to country. Factors affecting the comparison of reports of drug resistance in tuberculosis have been discussed by Horne. ${ }^{5}$ A difference has to be recognised between true primary resistance and undisclosed acquired resistance in patients who have concealed a history of previous chemotherapy. The two combined give a rate for initial drug resistance, and this varies widely from country to country. High rates, particularly to isoniazid, are recorded, for example, from Cairo and lower Egypt (34\%), India (26\%), Korea (24\%), and Thailand $(52 \%)$, and relatively low rates from Finland and Algeria (both $2 \%$ ). An average figure of $7 \%$ was recently recorded from 19 city and State laboratories in the United States, ${ }^{6}$ and one of $5 \%$ for Scotland. ${ }^{7}$ In a survey of 1038 isolates from patients in England and Wales in 1978-9 only $3 \%$ were resistant, $1.5 \%$ in whites and $7.5 \%$ in patients from the Indian subcontinent. ${ }^{8}$ Other studies have shown higher rates in immigrant populations. ${ }^{46}$ The overall rate is declining slowly in Britain.

The high rates of drug-resistant infections in some parts of the world are disquieting as a community problem. But what about the individual ? In patients infected with primary drugresistant organisms the disease responds well to chemotherapy provided that effective regimens are used-even in the absence of initial sensitivity testing. In a study in Hong Kong, where initial drug resistance is high, accurate tests of sensitivity before treatment gave surprisingly small benefits. Though $30 \%$ of patients in the study had initially drug-resistant organisms, quiescence was achieved in $89 \%$ of 187 patients when no notice was taken of the results of the sensitivity tests and in $92 \%$ when the regimen was adjusted to take account of the results of pretreatment tests. A quiescence rate of $95 \%$ was achieved in patients with fully sensitive organisms, though potentially the regimen used was $100 \%$ effective. ${ }^{9} 10$ In Britain only one out of every 200 patients with bacteriologically positive results who are given effective standard regimens would benefit from the routine use of accurate susceptibility tests. Such tests are, however, valuable in the intelligent management of patients with acquired resistance.

Short-course chemotherapy may be effective in the presence of initial drug resistance even to both isoniazid and streptomycin, provided that the regimen used includes streptomycin, isoniazid, rifampicin, and pyrazinamide for two months followed by rifampicin in the continuation phase. ${ }^{1112}$ When initial resistance to streptomycin, para-aminosalicylic acid, and isoniazid is frequent the drug regimen should contain both rifampicin and pyrazinamide, in part because of their special sterilising role and in part because initial resistance to them is infrequent. The level of resistance to rifampicin and pyrazinamide will, however, need to be watched with considerable interest and not a little anxiety in the next few years. If it is to remain low regimens will have to be used that largely overcome the influence of initial drug resistance. ${ }^{13} \mathrm{~A}$ disquieting survey of chemotherapy practices in 28 major metropolitan health departments in the United States has shown, however, that two-drug regimens (isoniazid with ethambutol or with rifampicin) were used most frequently in initial treatment; that only one-third of the programmes used three-drug regimens containing isoniazid, ethambutol, and rifampicin for initial treatment; and that only three of these used this regimen for more than $75 \%$ of their patients. ${ }^{14}$ 
Twenty years ago Canetti observed that "drug resistance is a function of the quality of chemotherapy which can be applied ... it is a problem of drug activity and drug acceptability. For many nations it is also a problem of cost." 15 The same is true today.

${ }^{1}$ Pyle MM. Relative numbers of resistant tubercle bacilli in sputa of patients before and during treatment with streptomycin. Mayo Clin Proc 1947; 22:465-73.

${ }^{2}$ Mitchinson DA. Basic mechanisms of chemotherapy. Chest 1979;76, suppl:771-81.

${ }^{3}$ Aswapokee P, Aswapokee N, Neu CO, Ellner PD, Neu HC. Drugresistant tuberculosis: serious problem. NY State 7 Med 1980;80: 1541-5.

${ }^{4}$ Kleeberg HH, Boshoff MS. $A$ world atlas of initial drug resistance. Paris: Scientific Committee on Bacteriology and Immunology of the International Union Against Tuberculosis, 1980.

5 Horne NW. Drug-resistant tuberculosis: a review of the world situation. Tubercle $1969 ; 50$, suppl:2-12.

- Anonymous. Primary resistance to anti-tuberculosis drugs-United States. Center for Disease Control. Morbidity and mortality weekly report $1980 ; 29: 345-6$.

7 Scottish Mycobacteria Reference Laboratory. Annual report. Scottish Mycobacteria Reference Laboratory, 1979.

${ }^{8}$ Medical Research Council Tuberculosis and Chest Diseases Unit. National survey of tuberculosis notifications in England and Wales, 1978-9. Br Med f 1980;281:895-8.

- Hong Kong Tuberculosis Treatment Services/British Medical Research Council Investigation. A study in Hong Kong to evaluate the role of pretreatment susceptibility tests in the selection of regimens of chemotherapy for pulmonary tuberculosis. Am Rev Respir Dis 1972;106:1-22.

${ }^{10}$ Hong Kong Tuberculosis Treatment Services/British Medical Research Council Investigation. A study in Hong Kong to evaluate the role of pretreatment susceptibility tests in the selection of regimens of chemotherapy for pulmonary tuberculosis-second report. Tubercle 1974 ;55: 169-92.

11 East African and British Medical Research Councils. Controlled clinical trial of five short-course (four month) chemotherapy regimens in pulmonary tuberculosis. First report of fourth study. Lancet 1978;ii: 334-8.

12 Singapore Tuberculosis Service/British Medical Research Council. Clinical trial of six-month and four-month regimens of chemotherapy in the treatment of pulmonary tuberculosis. Am Rev Respir Dis 1979;119: 579-85.

${ }^{13}$ Fox W. The modern management and therapy of pulmonary tuberculosis. Proc $R$ Soc Med 1977;70:4-15.

${ }^{14}$ Leff AR, Leff DR, Brewin A. Tuberculosis chemotherapy practices in major metropolitan health departments in the United States. $\mathrm{Am} \mathrm{Rev}$ Respir Dis 1981 ;123:176-80.

15 Canetti G. The eradication of tuberculosis: theoretical problems and practical solutions. Tubercle $1962 ; 43: 301-21$

\section{Children and parasuicide}

Prepubertal children rarely kill themselves, ${ }^{1}$ though between a tenth and a third of children under 13 years referred to psychiatric outpatient services ${ }^{2} 3$ admit to self-destructive thoughts, threats, or actions ("parasuicide"). ${ }^{4}$ Furthermore, some children admitted to hospital because of accidental poisoning may have deliberately taken the poison themselves. ${ }^{5}$ The incidence of parasuicide rises steeply in adolescence. In the United States suicide is the third most common cause of death in men between 15 and 24 years, ${ }^{6}$ and the rates are rising sharply among adolescents in Britain, ${ }^{7}$ though they are falling in other age groups.

The suicide rates for girls are much lower and have risen less, but self-destructive actions in girls outnumber those in boys by at least three to one. Around $10 \%$ of boys who attempt suicide eventually kill themselves ${ }^{8}$ and Shaffer found that at least $40 \%$ of the dead children had made previous threats. ${ }^{1}$ Parasuicidal behaviour in children must be taken seriously by clinicians.

Children who kill themselves come from disturbed back- grounds, are depressed, have close experience of suicidal behaviour in others, have access to effective means and an opportunity to use them, and have a degree of conceptual maturity which enables them to plan ahead. ${ }^{1}$ Parasuicidal behaviour, in contrast, is an impulsive act in a child who shares many of the features of the person who is successful in committing suicide but who is less likely to be depressed and more likely to come from a broken home. She may feel angry and resentful towards parents who have not met her basic needs for emotional care ${ }^{9}$ or have been abusive or rejecting. ${ }^{10}$ Children's concepts of death develop slowly, and some children harming themselves may possibly have the idea that death is temporary and "a pleasant state of pain cessation."

What are the causes of suicidal behaviour in prepubertal children ? The two major adult groups identified by factor analysis hold good for children too.11 Undoubtedly a few children have a depressive disorder, though child psychiatrists disagree about the nature and manifestations of prepubertal depression. ${ }^{12}{ }^{13}$ For far more children the action is likely to be a distorted and angry communication to a poorly functioning family. In adults parasuicide is recognised as both communicative and imitative ${ }^{14}$ : in children the same is true. In one study one-third of children taking overdoses knew of a relative or friend who had deliberately harmed himself. ${ }^{15}$ Other, non-specific, indicators of social stress are common (absent fathers, large families, low incomes, and delinquency). The threatened or actual loss of a parent may distinguish suicidal from non-suicidal adolescents. ${ }^{16}$

How can the incidence of parasuicide in prepubertal children be reduced? Primary prevention includes general measures to improve the wellbeing of children, including social support to poorly functioning families and improvement in the diagnosis and treatment of depression and other psychiatric disorders in childhood. Postgraduate tutors should increase the number of child psychiatric seminars in their programmes for general practitioners and hospital and community paediatricians. Courses for parents, teachers, and social workers would increase awareness in the community. Secondary prevention includes effective assessment and treatment for children presenting with parasuicidal behaviour and their families. All parasuicidal children should be admitted to the paediatric ward. The child psychiatric team should be available in every district general hospital to see a child of 16 or under within 48 hours of admission in order to use the crisis of admission therapeutically. Assessment must always include the whole family or other adults legally in charge of the patient. With training and consultant support non-medical members of the child psychiatric team can probably do much of the preliminary assessment, as is feasible in adults. ${ }^{17}$

Alternative treatment and management strategies in parasuicidal children have been little evaluated. Clinicians find that few patients need transfer to a child psychiatric ward; most can be managed on a paediatric ward for a few days and then as outpatients. Indications for transfer are the persistence of suicidal ideation or acts or the presence of florid psychotic symptoms. The treatment of choice is probably conjoint family therapy, focusing on the maladaptive family communication patterns and using techniques to promote change in the family system. ${ }^{18}$

Should psychotropic drugs be prescribed for depressed children? The vast increase in drug overdoses in adolescents has been mainly due to the ingestion of prescribed drugs, though not always drugs prescribed for the adolescent. General practitioners should rarely give psychotropic drugs to children without a specialist opinion, and drugs should be 